\title{
Non-fasting lipids and risk of cardiovascular disease in patients with diabetes mellitus
}

\author{
S. van Dieren • U. Nöthlings • Y. T. van der Schouw - A. M. W. Spijkerman • \\ G. E. H. M. Rutten - D. L. van der A • D. Sluik • C. Weikert • H. G. Joost • H. Boeing • \\ J. W. J. Beulens
}

Received: 19 July 2010 / Accepted: 20 September 2010 / Published online: 20 October 2010

(C) The Author(s) 2010. This article is published with open access at Springerlink.com

\begin{abstract}
Aims/hypothesis The aim of this study was to examine the effect of postprandial time on the associations and predictive value of non-fasting lipid levels and cardiovascular disease risk in participants with diabetes.

Methods This study was conducted among 1,337 participants with diabetes from the Dutch and German (Potsdam) contributions to the European Prospective Investigation into Cancer and Nutrition. At baseline, total cholesterol, LDL- and
\end{abstract}

S. van Dieren $(\bowtie) \cdot$ Y. T. van der Schouw $\cdot$ G. E. H. M. Rutten • J. W. J. Beulens

Julius Center for Health Sciences and Primary Care,

University Medical Center Utrecht,

Str. 6.131, P.O. Box 85500, 3508 GA Utrecht, the Netherlands

e-mail: S.vanDieren@umcutrecht.nl

U. Nöthlings $\cdot$ D. Sluik $\cdot$ C. Weikert $\cdot$ H. Boeing

Department of Epidemiology,

German Institute of Human Nutrition Potsdam-Rehbrücke,

Nuthetal, Germany

U. Nöthlings

Section of Epidemiology, Institute for Experimental Medicine, Christian-Albrechts University of Kiel,

Kiel, Germany

A. M. W. Spijkerman

Centre for Prevention and Health Services Research,

National Institute of Public Health and the Environment (RIVM),

Bilthoven, the Netherlands

D. L. van der A

Centre for Nutrition and Health,

National Institute of Public Health and the Environment (RIVM),

Bilthoven, the Netherlands

H. G. Joost

Department of Pharmacology,

German Institute of Human Nutrition Potsdam-Rehbrücke,

Nuthetal, Germany
HDL-cholesterol and triacylglycerol concentrations were measured and the ratio of total cholesterol/HDL-cholesterol was calculated. Participants were followed for incidence of cardiovascular disease.

Results Lipid concentrations changed minimally with increasing postprandial time, except for triacylglycerol which was elevated just after a meal and declined over time ( 1.86 at $0.1 \mathrm{~h}$ to 1.33 at $>6 \mathrm{~h}, p$ for trend $<0.001$ ). During a mean follow-up of 8 years, 116 cardiovascular events were documented. After adjustment for potential confounders, triacylglycerol (HR for third tertile compared with first tertile $\left(\mathrm{HR}_{\mathrm{t} 3 \mathrm{to} 1}\right), 1.73$ [95\% CI 1.04, 2.87]), HDLcholesterol $\left(\mathrm{HR}_{\mathrm{t} 3 \mathrm{tol}}, 0.41\right.$ [95\% CI $\left.\left.0.23,0.72\right]\right)$ and total cholesterol/HDL-cholesterol ratio $\left(\mathrm{HR}_{\mathrm{t} 3 \mathrm{tol}}, 1.65[95 \% \mathrm{CI}\right.$ $0.95,2.85])$ were associated with cardiovascular disease, independent of postprandial time. Cardiovascular disease risk prediction using the UK Prospective Diabetes Study risk engine was not affected by postprandial time.

Conclusions/interpretation Postprandial time did not affect associations between lipid concentrations and cardiovascular disease risk in patients with diabetes, nor did it influence prediction of cardiovascular disease. Therefore, it may not be necessary to use fasting blood samples to determine lipid concentrations for cardiovascular disease risk prediction in patients with diabetes.

Keywords Cardiovascular disease - Cholesterol . Coronary heart disease $\cdot$ Diabetes mellitus $\cdot$ Fasting lipids . Postprandial $\cdot$ Prediction $\cdot$ Triacylglycerol
Abbreviations
CVD Cardiovascular disease
EPIC European Prospective Investigation into Cancer and Nutrition
ICD International Classification of Diseases
UKPDS UK Prospective Diabetes Study 


\section{Introduction}

Guidelines recommend measuring fasting lipid profiles for cardiovascular disease (CVD) risk assessment [1]. The main reason for measurement of lipid concentrations during the fasting state is the possible increase in triacylglycerol levels up to $9 \mathrm{~h}$ after a meal. However, three different studies did not find a significant change in total cholesterol, HDL- or LDL-cholesterol or triacylglycerol over postprandial time [2-4]. This suggests that non-fasting lipid levels can be used to assess CVD risk in the general population.

However, this may be different for patients with diabetes. Cholesterol and triacylglycerol concentrations differ between patients with diabetes and people with normal glucose tolerance. Patients with diabetes tend to have higher total cholesterol and triacylglycerol levels and lower HDLcholesterol levels [5]. Furthermore, lipid responses after eating a meal in patients with diabetes are stronger compared with people with normal glucose tolerance [5]. So far, the effect of fasting time on the use of lipid levels for prediction of CVD risk in patients with diabetes has not been investigated.

Therefore, we examined the effect of time since last meal or drink on the associations of lipid levels and CVD risk in patients with diabetes. Furthermore, we assessed the effect of postprandial time on prediction of CVD risk in patients with diabetes using the UK Prospective Diabetes Study (UKPDS) risk engine [6].

\section{Methods}

The study population consisted of participants with diabetes from the Dutch and Potsdam (Germany) contributions to the European Prospective Investigation into Cancer and Nutrition (EPIC-NL and EPIC-Potsdam, $n=67,627$ ) [7, 8]. Both are observational cohort studies of the general population.

Participants from EPIC-NL were all confirmed diagnosed type 2 diabetes patients (total $n=387$ ), verified through medical records of the general practitioner or pharmacist [9]. In EPIC-Potsdam 265 patients were confirmed with type 2 diabetes and 685 patients had an unspecified diabetes type (total $n=950$ ), verified through repeated self-report in follow-up questionnaires.

In total 1,337 participants with diabetes at baseline were used for the analyses. Exclusion criteria were history of CVD $(n=239)$ and missing values on lipid concentrations $(n=169)$. All participants gave written informed consent prior to study inclusion. Both cohorts were approved by local ethical committees.

At baseline, participants filled out a general questionnaire regarding demographic characteristics and presence of chronic diseases. Body weight, height and blood pressure were measured. Postprandial time was defined as time since last meal or drink, which was recorded when the participants provided a blood sample. Total cholesterol, HDLand LDL-cholesterol and triacylglycerol concentrations were measured with the automatic ADVIA 1650 analyser (Siemens Medical Solutions, Erlangen, Germany) in EPICPotsdam and an auto-analyser (LX20; Beckman Coulter, Mijdrecht, the Netherlands) was used for the measurements of the cholesterol concentration for EPIC-NL participants. $\mathrm{HbA}_{1 \mathrm{c}}$ concentrations were measured in frozen erythrocytes using an immunoturbidimetric latex test.

Participants were followed for incidence of CVD using registries in the Netherlands [9] and follow-up questionnaires with subsequent verification in Germany. Diagnosis of CVD was coded according to the International Classification of Diseases (ICD; ICD-9 [www.icd9data.com/2007/Volume1/ 240-279/250-259/250/default.htm], ICD codes 410-414, 430-438; ICD-10 [www.who.int/classifications/icd/en/]; ICD codes I20-I25, I60-I67, I69).

Missing values for any of the confounders (ranging from $0.1 \%$ to $3.4 \%$ ) were imputed using single imputation.

Medians and interquartile ranges of lipid concentrations were examined across four categories of postprandial time $(0-2,2-4,4-6$ and $>6 \mathrm{~h})$ and plotted. Also categorical and continuous correlations between postprandial time and lipid concentrations were assessed.

The HR and 95\% CI were calculated for each tertile, with the lowest tertile as reference. Two different models were constructed. The first model was corrected for age, sex, smoking, systolic blood pressure, BMI, alcohol, $\mathrm{HbA}_{1 \mathrm{c}}$ concentration, duration since diagnosis of diabetes, energy intake, physical activity, diabetes medication and cohort. The second model was further adjusted for postprandial time as a continuous variable. Furthermore, HRs for standard deviation increase in lipid concentration were calculated across tertiles of postprandial time. Models were not adjusted for statin use because only two patients were using statins.

To investigate the difference in CVD risk prediction over fasting time, patients were divided into two groups, according to their postprandial time. A cut-off time between the two groups of $3 \mathrm{~h}$ was used because most patients had a postprandial time up to $3 \mathrm{~h}$ and previous studies have shown that lipid concentrations after eating a normal meal might be elevated for 3-4 h [3, 4].

The UKPDS risk engine was used to calculate 5 year CVD risk [6]. Discrimination was examined using the $c$ statistic. Calibration was examined using the HosmerLemeshow $\chi^{2}$ test.

\section{Results}

Baseline characteristics across tertiles of total cholesterol/ HDL-cholesterol ratio have been examined. Higher total 
cholesterol/HDL-cholesterol ratio was associated with a low education level (first tertile, 34\%; third tertile, $41 \%$ ), higher mean BMI (kg/m²) (first, 28.0; third, 30.2), mean systolic blood pressure (mmHg; first, 139; third, 143) and percentage $\mathrm{HbA}_{1 \mathrm{c}}$ (first, 7.74; third, 8.50). In total, 73.9\% of the participants were receiving either oral diabetes medication or insulin.

Only triacylglycerol concentrations declined over postprandial time, from $1.86 \mathrm{mmol} / \mathrm{l}$ at $0.1 \mathrm{~h}$ to $1.33 \mathrm{mmol} / \mathrm{l}$ at $>6 \mathrm{~h}$ ( $p$ value for trend $<0.001)$. While total cholesterol was $4.84 \mathrm{mmol} / 1$ at $0.1 \mathrm{~h}$ after a meal and $4.51 \mathrm{mmol} / \mathrm{l}$ after $>6 \mathrm{~h}$, HDL-cholesterol changed from 1.02 to $1.01 \mathrm{mmol} / \mathrm{l}$ and LDL-cholesterol from 2.83 to $2.79 \mathrm{mmol} / \mathrm{l}$ (Fig. 1).

Correlations between time since last meal or drink and lipid concentrations were only significant for triacylglycerols $(p<0.001$ for both continuous and categorical correlations). Continuous correlations between total cholesterol and postprandial time were significant $(p=0.04)$, while categorical correlations were not $(p=0.23)$

During a mean follow-up of 8 years, 116 incident cases of CVD were documented, of which 78 were coronary heart disease events. Increased triacylglycerol concentrations were associated with increased risk of CVD, while an inverse association was observed between levels of HDLcholesterol and CVD risk (Table 1). Ratio of total cholesterol/HDL-cholesterol was associated with an increased CVD risk. Further adjusting for postprandial time had no effect on the associations (model 2).
CVD HR for a standard deviation increase in lipid concentrations did not differ across tertiles of postprandial time (data not shown). The $p$ value for trend was $>0.08$ for all lipid concentrations.

Prediction of 5 year CVD risk calculated using UKPDS risk engine did not differ significantly between patients who had their lipid levels measured after a postprandial time of $>3 \mathrm{~h}(n=913)$ and $\leq 3 \mathrm{~h}(n=424)$. A discrimination of $0.71(95 \%$ CI $0.57,0.85)$ for $>3 \mathrm{~h}$ was only slightly better than $0.67(95 \%$ CI $0.59,0.75)$ for $\leq 3 \mathrm{~h}$. Calibration of the model was poor for both fasting-time durations ( $p$ value $<0.01$ for both time durations).

\section{Discussion}

In this cohort of 1,337 patients with diabetes, lipid concentrations did not change over postprandial time, except for triacylglycerols. Non-fasting lipid concentrations were associated with CVD risk independent of postprandial time. The associations between standard deviation of increase in lipid concentrations and CVD risk did not differ over postprandial time, which further strengthens the hypothesis that associations between lipid concentrations and CVD risk are not affected by postprandial time.

The strengths of this study are its relatively large sample size, validation of diabetes cases and geographical distribution of patients (from Germany and the Netherlands), but
Fig. 1 Median concentrations over postprandial time for concentrations of: (a) total cholesterol, $p=0.003$; (b) triacylglycerol, $p=0.001 ;(\mathbf{c})$ HDL-cholesterol, $p=0.23$; and (d) LDL-cholesterol, $p=0.88$. Error bars represent the interquartile range
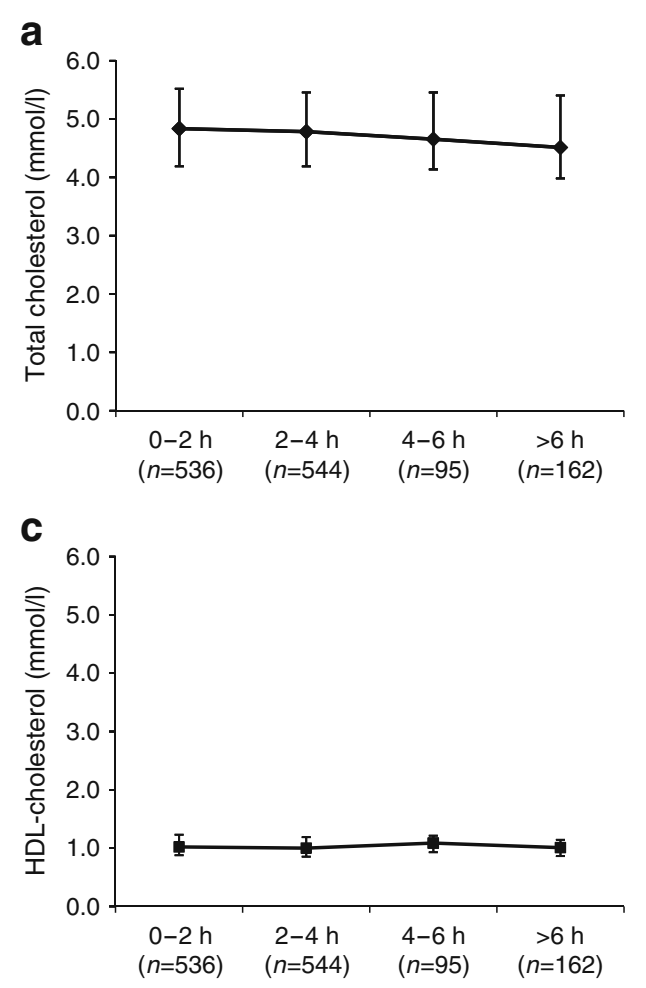
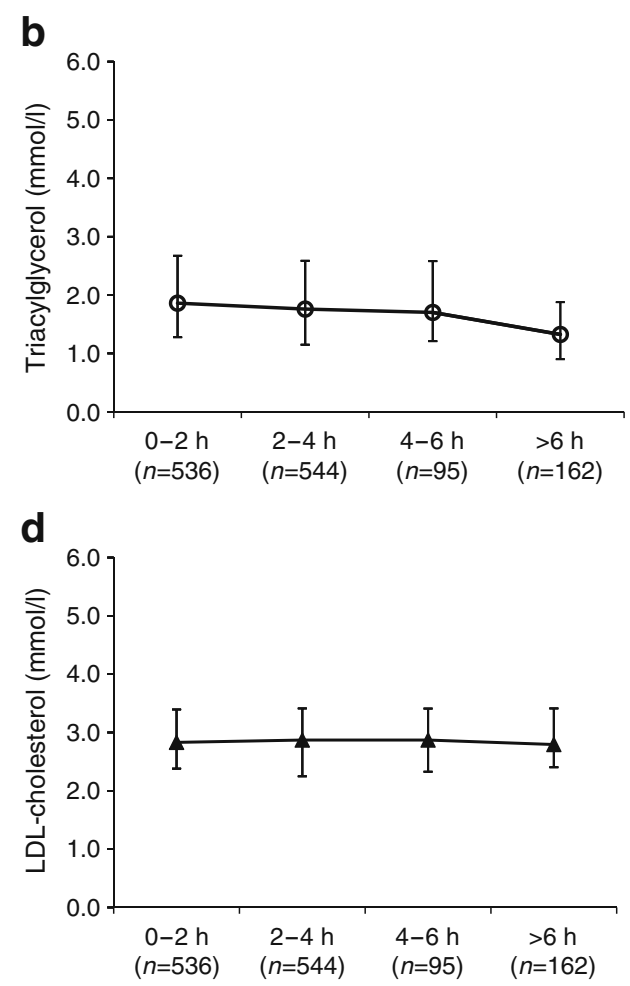
Table 1 HRs and 95\% CIs for CVD by tertile of lipid concentration in 1,337 patients with diabetes mellitus
Model 1 adjusted for age, sex, cohort, smoking, systolic blood pressure, $\mathrm{BMI}$, alcohol, $\mathrm{HbA}_{1 \mathrm{c}}$ level, duration since diagnosis of diabetes, energy intake, diabetes medication and physical activity

Model 2 adjusted for age, sex, cohort, smoking, systolic blood pressure, $\mathrm{BMI}$, alcohol, $\mathrm{HbA}_{1 \mathrm{c}}$ level, duration since diagnosis of diabetes, energy intake, diabetes medication, physical activity and postprandial time

\begin{tabular}{|c|c|c|c|}
\hline Variable & Cases $/ n$ & $\begin{array}{l}\text { Model } 1 \\
\text { HR }(95 \% \text { CI) }\end{array}$ & $\begin{array}{l}\text { Model } 2 \\
\text { HR }(95 \% \text { CI) }\end{array}$ \\
\hline \multicolumn{4}{|l|}{ Total cholesterol } \\
\hline Tertile $1(1.71-4.34)$ & $31 / 449$ & 1.00 & 1.00 \\
\hline Tertile $2(4.35-5.21)$ & $40 / 442$ & $1.15(0.71,1.85)$ & $1.16(0.72,1.87)$ \\
\hline Tertile $3(5.22-10.56)$ & $45 / 446$ & $1.05(0.64,1.73)$ & $1.05(0.64,1.73)$ \\
\hline$p$ value for trend & & 0.88 & 0.88 \\
\hline \multicolumn{4}{|l|}{ Triacylglycerols } \\
\hline Tertile $1(0.22-1.36)$ & $25 / 444$ & 1.00 & 1.00 \\
\hline Tertile $2(1.37-2.24)$ & $33 / 446$ & $1.09(0.64,1.87)$ & $1.11(0.65,1.90)$ \\
\hline Tertile $3(2.25-11.91)$ & $58 / 447$ & $1.73(1.04,2.87)$ & $1.79(1.07,2.98)$ \\
\hline$p$ value for trend & & 0.01 & 0.01 \\
\hline \multicolumn{4}{|l|}{ HDL-cholesterol } \\
\hline Tertile $1(0.40-0.91)$ & $53 / 445$ & 1.00 & 1.00 \\
\hline Tertile $2(0.92-1.14)$ & $44 / 438$ & $0.96(0.63,1.45)$ & $0.95(0.62,1.45)$ \\
\hline Tertile 3 (1.14-2.56) & $19 / 454$ & $0.41(0.23,0.72)$ & $0.41(0.23,0.72)$ \\
\hline$p$ value for trend & & 0.002 & 0.002 \\
\hline \multicolumn{4}{|l|}{ LDL-cholesterol } \\
\hline Tertile $1(0.54-2.48)$ & $37 / 459$ & 1.00 & 1.00 \\
\hline Tertile 2 (2.49-3.18) & $34 / 437$ & $0.79(0.49,1.28)$ & $0.79(0.49,1.28)$ \\
\hline Tertile 3 (3.19-6.62) & $45 / 441$ & $0.89(0.55,1.42)$ & $0.87(0.55,1.40)$ \\
\hline$p$ value for trend & & 0.65 & 0.61 \\
\hline \multicolumn{4}{|c|}{ Ratio total cholesterol/HDL-cholesterol } \\
\hline Tertile $1(1.73-4.15)$ & $21 / 445$ & 1.00 & 1.00 \\
\hline Tertile $2(4.16-5.27)$ & $42 / 446$ & $1.76(1.03,3.02)$ & $1.76(1.03,3.02)$ \\
\hline Tertile $3(5.28-13.82)$ & $53 / 446$ & $1.65(0.95,2.85)$ & $1.65(0.95,2.86)$ \\
\hline$p$ value for trend & & 0.14 & 0.14 \\
\hline
\end{tabular}

some limitations need to be addressed. First, the results may have been influenced by the limited number of patients with a postprandial time $>8 \mathrm{~h}$, which is the time limit for complete fasting. However, lipid concentrations did not change over postprandial time, therefore including more patients in complete fasting state would not have changed the results. Second, some differences in measurements between the cohorts may have occurred. However, the analyses were adjusted for the cohorts, and therefore it is unlikely that it affected the results.

Our findings among diabetes patients are in line with observations in the general population [2-4], although no association between CVD risk and LDL-cholesterol was found, as has been observed in other studies. Associations between triacylglycerols and HDL-cholesterol and CVD were stronger in our study compared with an earlier study by Langsted et al. [3]. These differences might be explained by differences in study population - our study focuses on diabetes patients whereas other studies focused on the general population.

Several studies have suggested that non-fasting lipids might predict CVD risk similarly to or even better than fasting lipids $[2,10]$, which is now confirmed by our study.
Therefore it may be unnecessary to measure fasting blood samples. Fasting requirements make blood sampling more burdensome for patients. It has been suggested that lipid levels might increase after a meal. Our study suggests that lipid levels do not change much in response to eating a normal meal, except for triacylglycerols. Moreover, postprandial time did not affect the ability to predict CVD risk in diabetes patients. This might be explained by the small contribution of lipids in CVD risk prediction models and therefore a slight increase in lipid concentrations after a meal may not substantially alter the risk [6].

In conclusion, postprandial time did not influence the association of lipid concentrations with CVD in patients with diabetes, nor did it affect the prediction of CVD risk. Therefore it may be unnecessary to use fasting blood samples to determine lipid concentrations for CVD risk prediction in patients with diabetes.

Acknowledgements This research was performed within the framework of the Center for Translational Molecular Medicine (CTMM; www.ctmm.nl), project PREDICCt (grant 01 C-104), and supported by the Netherlands Heart Foundation, Dutch Diabetes Research Foundation, Dutch Kidney Foundation and a European Foundation for the Study of Diabetes (EFSD)/sanofi-aventis grant. 
Duality of interest The authors declare that there is no duality of interest associated with this manuscript.

Open Access This article is distributed under the terms of the Creative Commons Attribution Noncommercial License which permits any noncommercial use, distribution, and reproduction in any medium, provided the original author(s) and source are credited.

\section{References}

1. De Backer G (2009) New European guidelines for cardiovascular disease prevention in clinical practice. Clin Chem Lab Med 47:138-142

2. Mora S, Rifai N, Buring JE, Ridker PM (2008) Fasting compared with nonfasting lipids and apolipoproteins for predicting incident cardiovascular events. Circulation 118:993-1001

3. Langsted A, Freiberg JJ, Nordestgaard BG (2008) Fasting and nonfasting lipid levels: influence of normal food intake on lipids, lipoproteins, apolipoproteins, and cardiovascular risk prediction. Circulation 118:2047-2056
4. Nordestgaard BG, Benn M, Schnohr P, Tybjaerg-Hansen A (2007) Nonfasting triacylglycerols and risk of myocardial infarction, ischemic heart disease, and death in men and women. JAMA 298:299-308

5. Chen YDI, Swami S, Skowronski R, Coulston A, Reaven GM (1993) Differences in postprandial lipemia between patients with normal glucose tolerance and noninsulin-dependent diabetes mellitus. J Clin Endocrinol Metab 76:172-177

6. Stevens RJ, Kothari V, Adler AI, Stratton IM (2001) The UKPDS risk engine: a model for the risk of coronary heart disease in type II diabetes (UKPDS 56). Clin Sci (Lond) 101:671-679

7. Boeing H, Wahrendorf J, Becker N (1999) EPIC-Germany-a source for studies into diet and risk of chronic diseases. European Investigation into Cancer and Nutrition. Ann Nutr Metab 43:195-204

8. Beulens JWJ, Monninkhof EM, Verschuren WMM, van der Schouw YT, Smit J, Ocke MC et al. (2010) Cohort profile: the EPIC-NL Study. Int J Epidemiol. doi:10.1093/ije/dyp217

9. Sluijs I, van der AD, Beulens JW et al (2010) Ascertainment and verification of diabetes in the EPIC-NL Study. Neth J Med 68:333-339

10. Eberly LE, Stamler J, Neaton JD (2003) Relation of triacylglycerol levels, fasting and nonfasting, to fatal and nonfatal coronary heart disease. Arch Intern Med 163:1077-1083 